\title{
Association between pretransplant serum soluble programmed death protein 1 level and prognosis following liver transplantation in patients with hepatocellular carcinoma
}

\author{
Kibong Oh${ }^{1}$, Shin Hwang ${ }^{2}$, Chul-Soo Ahn², Ki-Hun Kim², Deok-Bog Moon², Tae-Yong Ha², Gi-Won Song ${ }^{2}$, Dong-Hwan Jung ${ }^{2}$, \\ Kyung Jin Lee ${ }^{2}$, Eunyoung Tak ${ }^{2}$
}

\author{
${ }^{1}$ Department of Surgery, Anyang SAM Hospital, Anyang, Korea \\ ${ }^{2}$ Department of Surgery, Asan Medical Center, University of Ulsan College of Medicine, Seoul, Korea
}

Background: The study aimed to assess the prognostic influence of pretransplant serum soluble programmed death protein 1 (SPD-1) in patients undergoing liver transplantation (LT) for treatment of hepatocellular carcinoma (HCC).

Methods: Data from 229 patients with HCC who underwent living donor LT between January 2010 and December 2015 were retrospectively evaluated. Stored serum samples were used to evaluate SPD-1 concentrations.

Results: Tumor recurrence, overall survival, and HCC-specific survival rates were $25.5 \%, 94.3 \%$, and $96.0 \%$ at 1 year; $40.8 \%$, $78.2 \%$, and $80.7 \%$ at 3 years; and $44.5 \%, 75.4 \%$, and $77.9 \%$ at 5 years, respectively. Prognostic analysis using pretransplant serum SPD-1 with a cutoff of $93.6 \mu \mathrm{g} / \mathrm{mL}$ (median value of the study cohort) did not have significant prognostic influence on HCC recurrence, HCC-specific patient survival and post-recurrence patient survival $(P \geq 0.26)$. Prognostic analysis using SPD-1 with a cutoff of $300 \mu \mathrm{g} / \mathrm{mL}$ showed marginally higher tumor recurrence $(P=0.069)$, similar HCC-specific patient survival $(P=0.25)$ and higher post-recurrence patient survival $(P=0.045)$. Multivariate analysis revealed that Milan criteria were prognostic for HCC recurrence and HCC-specific patient survival, but pretransplant SPD1 with a cutoff of $300 \mu \mathrm{g} / \mathrm{mL}$ did not become an independent prognostic factor.

Conclusions: The results of this study demonstrate that pretransplant serum SPD-1 may not have significant influences on posttransplant outcomes in patients with HCC, although there might be some potential prognostic influence from very high expression of serum SPD-1. Additional large-scale, multicenter studies and detailed mechanism studies are required to clarify the role of serum SPD-1 in LT recipients. 